\title{
Journal of Acute Disease
}

journal homepage: www.jadweb.org

Case report https://doi.org/10.12980/jad.6.2017JOAD_2016_51

(C2017 by the Journal of Acute Disease. All rights reserved.

\section{Acute Guillain-Barré syndrome: A case report in pediatrics}

Charishma Modupalli, Chandrakala Sriramulu, Divya Gopineni", Prasanna Raju Yalavarthi, Ranganayakulu Diviti

Pharmacy Practice Division, Sri Padmavathi School of Pharmacy, Tiruchanur 517503, Tirupati, India

\section{ARTICLE INFO}

Article history:

Received 24 Aug 2016

Received in revised form 31 Aug 2016

Accepted 12 Oct 2016

Available online 2 Dec 2016

Keywords:

Areflexia

Hypotaxia

Hypotonia

Immunoglobulins

Neuropathy

Sensory loss

Tachypnea

\section{ABSTRACT}

Guillain-Barré syndrome is a complicated degenerative neurological disorder which can be either acute or chronic in nature. Guillain-Barré syndrome is an acquired condition and is characterized by progressive, symmetrical, proximal and distal tingling and weakness. Muscle stretch reflexes are decreased to absent and loss of sensation is common. A 4-year female child with no significant past medical history who presented with progressive weakness involving both upper and lower limbs over 48 to $72 \mathrm{~h}$ and complaints of tachypnea and hypotonia involving all group of muscles was admitted in Sri Venkateswara Ramnarayan Ruia Government General Hospital, Tirupati. In the present report, the main clinical aspects and features of Guillain-Barré syndrome along with the multidisciplinary approach to the acute phase combining supportive and high dose of immunoglobulin therapy were practiced. Patient should have follow-up within 2 weeks after the acute syndrome to evaluate for relapse, at which point repeat intravenous immunoglobulin. Thereafter, follow-up is necessary for every 4-6 weeks for 6 months, then 6 months for 1 year and then yearly.

\section{Introduction}

Guillain-Barré syndrome or acute idiopathic polyradiculoneuritis is a rare and serious immune-mediated peripheral neuropathic disorder[1]. Guillain-Barré syndrome is manifested with progressive proportionate weakness as well as loss of sense. The annual incidence rate for Guillain-Barré syndrome according to the National Institute of Neurological Disorders is about $1-2$ in 100000 population. Approximately, 100 years ago, the disease Guillain-Barré syndrome was first coined by French physicians Guillain, Barre and Strohl. It is a complicated degenerative neurological disorder which can

*Corresponding author: Divya Gopineni, Pharmacy Practice Division, Sri Padmavathi School of Pharmacy, Tiruchanur 517503, Tirupati, India.

Tels: 09573566460 (C Modupalli); 09491288619 (D Gopineni)

E-mails: charishmamodupalli@rocketmail.com (C Modupalli); divyagopineni@ gmail.com (D Gopineni)

The journal implements double-blind peer review practiced by specially invited international editorial board members. be acute or chronic in nature. In the acute phase of GuillainBarré syndrome, approximately $3 \%$ of patients may die from acute complications and up to $20 \%$ of patients have residual, permanent and severe disability with ambulation deficits[2].

The underlying etiology and pathophysiology of GuillainBarré syndrome are not completely and clearly understood, but it is thought to be an immune-mediated process, resulting from the generation of autoimmune antibodies and inflammatory cells that cross-reacts with antigenic determinant on peripheral nerves and roots, leading to demyelination (loss of myelin) and/or axonal damage. This immune response is thought to be initiated in response to a variety of antigenic stimuli, such as viral or bacterial infection (particularly Campylobacter jejuni) [3]. The main types of Guillain-Barré syndrome are acute inflammatory demyelinating polyradiculoneuropathy, MillerFisher syndrome, acute motor axonal neuropathy and acute motor-sensory axonal neuropathy[2,4,5]. 
Signs and symptoms of Guillain-Barré syndrome are prickling, sensations of "pins and needles" in fingers, toes, ankles or wrists, weakness in legs that spreads to upper body, unsteady walking or inability to walk, difficulty with eye or facial movements, including speaking, chewing or swallowing, severe pain that may feel achy or cramp and may be worse at night, difficulty with bladder control or bowel function, rapid heart rate, low or high blood pressure, difficulty breathing, muscle weakness, loss of reflexes and numbness or tingling in arms, legs, face and other parts of body[4,5]. This rare condition could result in paralyze and may lead to death at times.

Guillain-Barré is difficult to diagnose at first because the symptoms are very similar to those of other neurological disorders or conditions that affect the nervous system, such as botulism, heavy metal poisoning or meningitis. Diagnostic tests include spinal tap, electromyography and nerve conduction test[5,6]. Differential diagnoses of Guillain-Barré syndrome were conducted when the diagnostic tests alone can't confirm the disease which includes chronic inflammatory demyelinating polyneuropathy, myasthenia gravis and voluntary muscle weakness. During the clinical investigation, infection may be characterized by cerebrospinal fluid cell count, spinal abnormalities, radicular pain, activity-induced pains, physical state of consciousness, intolerable headache and loss of motor and sensory actions.

Treatments of Guillain-Barré syndrome include plasma exchange (plasmapheresis), intravenous immunoglobulin (high doses of immunoglobulin can also help to block the antibodies causing Guillain-Barre), pain relieved medications, and also treatments to prevent blood clots for immobile patients. Steroids are also used for the treatment of acute Guillain-Barré syndrome[7-9].

\section{Case report}

A female child with the age of 4 years old was admitted in the private hospital due to progressive weakness, including bulbar muscles over 48 to $72 \mathrm{~h}$ following a trivial injury i.e., falling from bed. Weakness then increased rapidly during the day involving both upper and lower limbs and she was transferred to Sri Venkateswara Ramnarayan Ruia Government General Hospital, Tirupati. On admission child patient was conscious and coherent and had complaints of tachypnea (decreased effort of respiration), hypotonia involving all group of muscles (lower and upper limbs, trunk, neck) and hypotaxia. Neurological abnormalities including reduced power (both lower and upper limbs i.e., $0 / 5$ and $1 / 5$, respectively) and deep tendon reflexes were absent (areflexia). Cranial nerves and sensations all over the body were intact. Her nerve conduction showed an acute motor axonal neuropathy variant of Guillain-Barré syndrome.

During the examination at the emergency unit, she had no fever, respiratory tract infection or gastroenteritis. She had no exposure to toxins and poison, or had no recent vaccination and no loss of appetite. She was rational but had flaccid quadriplegia with areflexia and flexure plantar response. The right median ulnar and bilateral posterior tibial motor conductions show normal distal patency and conduction velocity with portion amplitude of grossly reduced compound muscle action and absent f-wave. The evoked motor response was absent from bilateral common peroneal nerve. Sensory conductions were not within normal limits.

One month back she had suffered with fever for eight days. In the second half of that period (about four days) she was unable to walk without support and had marked difficulty in getting up from sitting position and she suddenly fell from bed and gradually developed weakness of both lower limbs progressing over a period of 42 to $72 \mathrm{~h}$. She was hemodynamically stable with pulse rate of $120 / \mathrm{min}$, and blood pressure was decreased to 94/60 mmHg with elevated body temperature $37.7^{\circ} \mathrm{C}$. Her basic laboratory investigations were all in normal except hematocrit (23.3\%) indicating anemia condition.

A diagnosis of Guillain-Barré syndrome had been made. She was treated with intravenous immunoglobulin for 10 days along with various vitamin supplements without requiring ventilator support. At the day 8 of hospitalization, the patient tolerated liquid every $4 \mathrm{~h}$ via gavage tube, as well as $5 \mathrm{~mL}$ of Bevon syrup once daily. And $30 \mathrm{~g}$ of protein powder in one glass of milk, 100000 IU of vitamin A syrup and $5 \mathrm{~mL}$ of zinc syrup once daily were prescribed. She gradually improved over the next one month and discharged with vitamin supplements i.e., vitamin $\mathrm{A}$ and $\mathrm{D}$ capsules, vitamin $\mathrm{C}$ and protein powder.

\section{Discussion}

Guillain-Barré syndrome is an acute areflexic polyneuropathy of autoimmune nature. The weakness reaches its peak within 4 weeks. Guillain-Barré syndrome is considered to be a monophonic disease and subtype neurophysiology with antiganglioside auto antibodies such as acute inflammatory demyelinating polyneuropathy, acute motor axonal neuropathy, acute motor sensory axonal neuropathy and Miller- Fisher variant of Guillain-Barré syndrome[10].

Both acute motor axonal neuropathy and acute inflammatory demyelinating polyneuropathy subtypes of Guillain-Barré 
syndrome occur frequently in children admitted to hospitals and findings suggest that acute motor axonal neuropathy is an important subtype of Guillain-Barré syndrome seen in pediatric population. In severe cases, such as in the acute motor axonal neuropathy or acute motor sensory axonal neuropathy variants, axonal degeneration occurs, and recovery depends on axonal regeneration. Recovery becomes slower, and there is a greater degree of residual damage. According to neurophysiological criteria for acute motor sensory axonal neuropathy form of Guillain-Barré syndrome, the sensory potentials are either absent or markedly reduced[11].

In some previous instances, treatment of Guillain-Barré syndrome by combination of plasma exchange and intravenous immunoglobulin has been shown its promise. The choice between them is often hospital dependent. Intravenous immunoglobulin causes risk of transmitting infections. In such case, antibiotics are prescribed which may worsen the situation by inhibiting auto-antibodies. IV immunoglobulins also block the binding of Fc gamma receptors, preventing phagocyte damage from macrophages[12]. Studies have shown that an optimal amount of IV immunoglobulins is $400 \mathrm{mg}$ / $\mathrm{kg}$ administered over 6 days. It is, however, much easier to administer because it is a peripheral intravenous infusion. In this patient, nutrition support was suggested because the maintenance of fluid and electrolyte balance, immune competence, prevention of superimposed starvation-induced wasting of the muscles from the effects of metabolic stress. Such maintenance could be possible through feeding sufficient amounts of nutrients and optimal distribution of non-protein calories. If optimal nutritional support was provided, that may lead to any hypermetabolic/catabolic state, including infectious complications and failure to wean from mechanical ventilation.

\section{Conclusion}

Guillain-Barré syndrome is a neurological disorder resulting primarily in muscle paralysis, which in most cases dissymmetrical patient may have mild involvement or severe involvement which may in a small percentage lead to death. Guillain-Barré syndrome is rare. However, it must be considered in the differential diagnosis of any patient who presents with progressive weakness. It is important to recognize the variety and severity of the neurological symptoms associated with Guillain-Barré syndrome, especially with the diagnostic difficulties associated with the pediatric population. Patient should have follow-up within 2 weeks after the acute syndrome to evaluate for relapse, at which point repeat intravenous immunoglobulin. Thereafter, followup is necessary for every 4 to 6 weeks for 6 months, then 6 months for 1 year and then yearly. Patient should continue working with physiotherapy and occupational therapy. Patients should be instructed to contact their physician with any worsening symptoms of weakness, numbness, paraesthesia, facial weakness and difficulty with swallowing or breathing, or worsening bladder function.

\section{Conflict of interest statement}

The authors report no conflict of interest.

\section{References}

[1] Sebastian S. A case of Guillain-Barré syndrome in a primary care setting. J Nurse Pract 2012; 8(8): 643-8.

[2] Sejvar JJ, Baughman AL, Wise M, Morgan OW. Population incidence of Guillain-Barré syndrome: a systematic review and meta-analysis. Neuroepidemiology 2011; 36: 123-33.

[3] Mazidi M, Imani B, Norouzy A, Rezaie P. Guillain-Barré syndrome: a case report. Int J Hosp Res 2013; 2(2): 91-3.

[4] Pithadia AB, Kakadia N. Guillain-Barré syndrome (GBS). Pharmacol Rep 2010; 62: 220-32.

[5] Newswanger DL, Warren CR. Guillain-Barré syndrome. Am Fam Physician 2004; 69(1): 2405-10.

[6] Winer JB. Treatment of Guillain-Barré syndrome. QJM 2002; 95: $717-21$.

[7] Ghabaee M, Ghanbarian D, Brujeni GN, Bokaei S, Siavoshi F, Gharibzadeh S. Could Helicobacter pylori play an important role in axonal type of Guillain-Barré syndrome pathogenesis. Clin Neurol Neurosurg 2010; 112(3): 193-8.

[8] Wadasingha DL, Weerawansa MRP, Siribaddana SH. Recurrent Guillian-Barré syndrome - a case report. Anuradhapura Med J $2015 ; 9(1): 15-7$

[9] Aquil N, Khan IA, Soomro B. Guillian Barré syndrome in a family: a case report of four siblings. J Coll Physicians Surg Pak 2011; 21(3): 179-81.

[10] Fokke C, van den Berg B, Drenthen J, Walgaard C, van Doorn PA, Jacobs BC. Diagnosis of Guillian-Barré syndrome and validation of Brighton criteria. Brain 2014; 137: 33-43.

[11] Rooper AH. The Guillian-Barre syndrome. N Engl J Med 1992; 326(17): 1130-6.

[12] Lasky T, Terracciano GJ, Magder L, Koski CL, Ballesteros M, Nash D, et al. The Guillian-Barre syndrome and the 1992-1993 and 1993-1994 influenza vaccines. N Engl J Med 1998; 339(25): 1797-802. 